\title{
Fronts pionniers d'Amazonie. Les dynamiques paysannes au Brésil
}

\section{Eric Sabourin}

L'ouvrage issu de la thèse de géographie de Xavier Arnauld de Sartre porte sur les dynamiques de reproduction des unités d'exploitation familiales d'une agriculture paysanne sur les fronts pionniers de l'Amazonie Brésilienne. Les travaux ont été conduits chez des agriculteurs établis au long de la route la transamazonienne dans la région d'Altamira (Etat du Para) en collaboration avec les équipes de l'Université Fédérale du Pará (Département d'études intégrées sur l'agriculture familiale à Belém et Laboratoire Agroécologique de la Transamazonienne à Altamira). Le livre est divisé en cinq chapitres qui traitent successivement des transformations des dynamiques de gestion des ressources et du travail familial en partant du cadre de la politique de colonisation agricole au Brésil (chapitre 1) pour terminer sur la question clef de la caractérisation des logiques et des itinéraires de l'actuelle génération de jeunes paysans et fils d'agriculteurs (chapitre V).

Les enjeux et défis de la reproduction des exploitations agricoles familiales sont analysés autour des jeunes agriculteurs identifiés eux-mêmes par l'auteur comme « objet frontière » sur la frontière de colonisation agricole.

L'auteur voit dans le processus de construction identitaire des jeunes agriculteurs l'émergence d'un sujet, acteur clef des dynamiques de développement local et de ruralisation des fronts pionniers amazoniens, pourtant construits autour de petites villes au long de la route fédérale.

L'analyse sociologique des familles paysanne permet à Xavier Arnauld de Sartre de caractériser le processus de subjectivisation des jeunes agriculteurs à partir de la confrontation entre l'héritage familial (issu d'une tradition paysanne) et les confrontations du milieu écologique, économique et sociopolitique. Bien que Touraine ne soit pas mobilisé, on trouve la une singulière actualisation de ses thèses sur la construction du sujet et le rôle du mouvement social dans un contexte de ruralité latino-américaine. "C'est parce qu'ils sont devenus des personnes conscientes des contraintes qui pèsent sur eux et des limites de leur environnement, des sujets qui veulent agir depuis un milieu particulier pour le transformer et se transformer eux-mêmes, que les changements que l'on observe sont possibles et qu'un monde rural peut émerger dans la région - .p209»

Mais la contribution la plus originale de l'ouvrage provient de la mobilisation au bénéfice de la géographie humaine des théories anthropologiques et sociologiques du don et de la réciprocité afin de caractériser la diversité des logiques sociales de gestion du milieu et des trajectoires d'exploitations familiales sur les fronts pionniers. Premièrement, la typologie des agriculteurs familiaux sur les fronts pionniers mobilise les modalités dynamiques et dialectiques (et non pas seulement structurelles) 
du lien social (parenté/alliance, proximité/mobilité, entraide/autonomie, etc.) comme un critère essentiel.

Deuxièmement, A. de Sartre a recours aux catégories du don, contre-don et de la dette (Mauss, 1924 ; Bourdieu, 1994) pour expliquer les dynamiques familiales entre génération et en particulier les logiques paternalistes et communautaires. Pour les types «reproduction du paternalisme paysan» et "paternalistes paysans intermédiaires » le paternalisme familial destiné à assurer un retour des enfants aux parents revêt le caractère de l'exploitation paternaliste, asymétrique ou inégalitaire, déjà identifiée au Brésil (Léna, Geffray \& Araujo, 1996) : le don de vie et le don de la terre des parents implique une dette et une obligations d'aide des enfants, soient via des emplois salariés de type urbain, soit par la reproduction de l'unité familiale agricole des parents. Le type «paysans communautaires » mobilise des relations plus symétriques entre générations ou la proximité physique et sociale importe plus que l'aide matérielle.

Mais dans les deux cas le recours des jeunes au marché du travail salarié peut constituer ou bien un élément de continuité de la solidarité ou une occasion de rupture de la domination et la dépendance vis-à-vis des parents. Cela dépend des valeurs humaines ou éthiques associées au projet paysan ou au projet communautaire local.

Il est dommage qu'A de Sartre n'ait pas poursuivi cette analyse en termes de réciprocité à partir des catégories de Polanyi. La réciprocité comme principe économique et social caractérise précisément le redoublement d'une action entre sujets et entre autre la reproduction des dons ou des dettes. La réciprocité peut être symétrique (horizontale, entre pairs) ou asymétrique (cas de l'aliénation paternaliste). Temple (1998, 2004) a montré que la récurrence de ces relations dessine des structures de réciprocité qui peuvent être binaires (face a face entre individus ou entre groupes) ou ternaires (incluant un tiers sujet) et que chaque structure de réciprocité élémentaire engendre à la fois des prestations matérielles (valeurs d'usage) et des valeurs éthiques spécifiques. Les liens entre générations constituent des relations de réciprocité ternaires (unilatérales) qui produisent précisément la responsabilité. La gestion partagée des ressources naturelles (terres, forêt, eau) correspond à une structure de réciprocité ternaire engendrant, en plus des valeurs d'usage, la confiance et la justice entre les membres du groupe.

La théorie de la réciprocité permet de surmonter les limites de l'opposition entre le don et le marché, par une relation dialectique, en complémentarité ou en contradiction, entre principe de réciprocité et principe d'échange. Elle explique, entre autre comment le recours à l'échange marchand (du travail, des biens ou des savoirs) s'il en vient à dominer le principe de réciprocité, élimine ou annihile la production de valeurs éthiques, privilégiant les seuls intérêts matériels individuels.

Le recours à l'échange marchand (salariat des jeunes), sans contrôle des structures de réciprocité familiales, communautaires ou citoyennes, peut apparaître à juste titre comme une libération de l'oppression paternaliste, mais il risque également 
d'introduire l'exploitation capitaliste et la concurrence pour l'accumulation individuelle.

C'est la que le rôle régulateur de l'Etat (local, régional ou fédéral), souvent le grands absent des fronts pionniers, est essentiel que ce soit vis a vis des marchés d'échange, du respect de la législation du travail ou surtout de l'accès aux ressources naturelles et immatérielles (santé, éducation). Les acquis communautaires des paysans amazoniens et les limites des ressources et des compétences publiques devraient donc inciter à privilégier des interactions entre les politiques publiques et l'action collective des ruraux. Plus que les financements externes, la reconnaissance publique et juridique des dispositifs collectifs et institutionnels des agriculteurs assurant la gestion de ressources communes (terres, forêt, eau, biodiversité) ou la production de biens publics (éducation, innovation, information, savoir faire). Le cas des Maisons familiales rurales est exemplaire, comme celui des réserves extractivistes ou de certains projets environnementaux et de réforme agraire. Ces dispositifs sont destinés à appuyer la production familiale ou individuelle (en partie destinée au marché d'échange) mais fonctionnent sur la base de prestations gratuites de leurs membres, c'est-à-dire a partir de relations de réciprocité entre pairs. Les paysans amazoniens, comme ceux d'autres régions du Brésil (Caron et Sabourin, 2001) ou du monde, ont su construire des interfaces entre systèmes de réciprocité et système d'échange.

Septembre 2007, Éric Sabourin Cirad - UR Action collective, politiques et marchés Université de Brasilia

Xavier Arnauld de Sartre 2006, Fronts pionniers d'Amazonie. Les dynamiques paysannes au Brésil. Paris, CNRS editions, col. Espaces \& Milieux, 222p Préface de H Théry ISBN 2-271-06441-4, 27 euros.

\section{Références}

Bourdieu, P. 1994 Raisons pratiques. Sur la théorie de l'action. Paris, Le seuil, 248p.

Caron, P., Sabourin, E (Coord) 2001 Paysans du Sertão. Mutations des agricultures familiales dans le Nordeste du Brésil. Cirad, Embrapa, Montpellier, coll. Repères, $243 p$

Lena, P.; Geffray, C.; Araujo, R. (direct.) (1996) L'oppression paternaliste au Brésil. In: Lusotopie 1996. Karthala/CEAN, Paris, pp.105-354

Mauss M, [1924] 1950. "Essai sur le Don", In: Sociologie et Anthropologie, Paris, PUF, 7ème édition, réédition 1989, PUF collec. Quadrige.

Polanyi K, Arensberg C, 1975, Les systèmes économiques dans l'histoire et dans l'économie, París, Librairie Larousse. Ed. originale [1957] Trade and Market in the Early empires.

Temple, D. 1998. Les structures élémentaires de la réciprocité Revue du MAUSS n¹2, (2) : 234-242,

Temple, D. Teoria de la Reciprocidad. La Paz: PADEP/ GTZ, 2004, 3 tomes 